\title{
Les nouvelles normes de croissance de l'OMS
}

\section{Comparaison à d'autres courbes de croissance}

\author{
Ekhard E. Ziegler Steven E. Nelson \\ Service de Pédiatrie, Université de l'lowa, lowa City, lowa, États-Unis
}

\section{Mots-clés}

Normes de croissance de l'OMS · Courbes de croissance

\section{Résumé}

Les nouvelles normes de croissance de l'Organisation mondiale de la Santé (OMS) ont été créées dans l'intention d'obtenir des normes de croissance globalement applicables décrivant la croissance des enfants telle qu'elle se déroule dans des conditions nutritionnelles optimales et en l'absence de contraintes externes. En d'autres termes, elles représentent la croissance telle qu'elle devrait être. Les données destinées à la création des nouvelles normes ont été recueillies chez des enfants de six pays vivant dans des circonstances favorables. De plus, les données de certains sujets se situant à des valeurs extrêmes de poids et de taille ont été exclues. De façon non surprenante, les nouvelles normes de croissance diffèrent de plusieurs façons des autres courbes de croissance de référence. Ces différences étant nombreuses, seules quelques remarques générales sont possibles. (1) Au cours des dix premiers mois de la vie, les poids et tailles indiqués par les normes de l'OMS sont plus élevés à tous les percentiles que ceux indiqués par toute autre courbe de référence. (2) Au cours du deuxième semestre de la vie et jusqu'à l'âge de deux ans, le poids mentionné par les normes de l'OMS (mais non la taille) est plus bas que celui indiqué par les autres courbes. (3) Entre 2 et 5 ans, le poids de référence de I'OMS tend à se situer à l'extrémité inférieure du spectre, particulièrement aux plus bas percentiles, tandis que la courbe EuroGrowth occupe l'extrémité supérieure pour le poids à tous les

\section{KARGER}

Fax +41613061234 E-Mail karger@karger.ch www.karger.com
(C) 2008 Nestec Ltd., Vevey/S. Karger AG, Basel 0250-9644/07/0653-0111\$23.50/0

Accessible en ligne à:

www.karger.com/anf percentiles. (4) Une évaluation fonctionnelle a montré que, comparativement à d'autres courbes de référence, les normes de l'OMS identifiaient moins d'enfants de 1 à 5 ans comme étant en souspoids et un plus grand nombre d'enfants de 2 à 5 ans comme étant en surpoids.

Copyright $\odot 2008$ Nestec Ltd., Vevey/S. Karger AG, Basel

\section{Introduction}

Les courbes de croissance de référence sont des outils importants pour l'évaluation de la croissance et de l'état nutritionnel d'un enfant particulier (utilisation clinique) et de groupes d'enfants (utilisation épidémiologique). Ces courbes sont perçues comme représentant la croissance normale de l'enfant et ont donc une autorité normative. De nombreux pays disposent de courbes nationales de croissance, mais un très grand nombre n'en ont pas. Des courbes universelles de croissance sont nécessaires dans ces pays, et également afin de faciliter des comparaisons internationales. Afin de répondre à ce besoin, l'Organisation mondiale de la Santé (OMS) a adopté en 1978 des courbes de croissance qui avaient été construites par le Centre national des statistiques de santé (National Center for Health Statistics; NCHS) des ÉtatsUnis [1]. Ces courbes sont connues sous le nom de courbes de croissance NCHS/OMS.

Il est cependant bientôt apparu qu'elles ne représentaient pas de façon très fidèle la croissance des nourrissons. Com- 
parativement au poids des nourrissons au sein en Europe et aux États-Unis [2], le poids pour l'âge indiqué par les courbes NCHS/OMS s'est avéré trop faible au cours des six premiers mois de la vie, alors qu'il tendait à être plus élevé au cours du second semestre [3]. Ces inconvénients ont été principalement attribués à deux caractéristiques de l'étude sous-jacente de la croissance. Premièrement, les données anthropométriques concernant la première année de la vie étaient trop espacées (tous les trois mois). Deuxièmement, peu de nourrissons ont été exclusivement allaités au sein, et parmi ceuxci, un grand nombre l'ont été pendant une courte durée. Le fait que les sujets vivaient dans une seule zone géographique des États-Unis a été également considéré comme un inconvénient. Un comité d'experts réunis par l'OMS a conclu que les courbes NCHS/OMS étaient inappropriées et inadéquates, et a recommandé la création de nouvelles courbes pour une utilisation générale [3]. Une étude multicentrique destinée à déterminer la croissance de référence a été mise en place et des données ont été recueillies de 1997 à 2003 pour la création de nouvelles normes représentatives des enfants de la naissance à 5 ans au plan international. Les nouvelles normes de croissance de l'OMS pour une utilisation générale ont été publiées en 2006 [4].

Les courbes de référence ont traditionnellement décrit la croissance d'enfants considérés comme en bonne santé et qui vivaient dans une zone géographique définie, qu'il s'agisse d'un seul pays comme dans le cas des courbes du NCHS [1], ou d'un groupe de pays comme dans celui des courbes EuroGrowth [5]. Ces courbes sont appelées courbes de croissance de référence. Les nouvelles normes de croissance de l'OMS s'écartent de cette tradition et ne décrivent pas simplement la croissance d'enfants en bonne santé. Au contraire, elles visent à représenter la croissance optimale des enfants. Au plan opérationnel, cette croissance a été définie comme celle d'enfants vivant dans des conditions ne lui imposant aucune contrainte. Dans le cas des nourrissons et des jeunes enfants, ces conditions impliquent une nutrition conforme aux recommandations de l'OMS. Les courbes de croissance de l'OMS sont donc classées comme des «normes» distinctes des «références» qui décrivent la croissance de tous les enfants, y compris de ceux ne vivant pas dans des conditions favorables. Les normes de l'OMS visent également à être globalement représentatives au plan international.

Deux nouvelles courbes de référence ont été publiées pendant le temps de la création des nouvelles normes de l'OMS. Les courbes Euro-Growth, résultant d'une étude de la croissance d'enfants à l'échelon européen [5] ont été publiées en 2000. Également en 2000, les Centers for Disease Control and Prevention (CDC) ont publié de nouvelles courbes de croissance basées sur les États-Unis [6,7] et qui résultaient d'une révision des courbes du NCHS entreprise dans l'intention de corriger leurs inconvénients.

Nous présentons ici les nouvelles normes de croissance de l'OMS par rapport aux courbes NCHS/OMS, qu'elles ont rem- placées, et également aux courbes Euro-Growth et des CDC. Nous verrons par quels aspects les normes de l'OMS diffèrent des autres courbes et également comment ces dernières diffèrent entre elles. Un grand nombre de ces différences sont trop faibles pour exercer une influence quelconque, mais certaines sont assez importantes et ont probablement des conséquences fonctionnelles.

\section{Les nouvelles normes de croissance de I'OMS}

Une étude multicentrique destinée à déterminer la croissance de référence (étude MGRS) a été menée de 1997 à 2003 avec pour objectif de décrire la croissance d'enfants vivant dans des conditions ne lui imposant aucune contrainte. L'intention était de créer des normes représentatives au plan international, et des données ont donc été recueillies dans six centres de six pays (Brésil, Ghana, Inde, Norvège, Oman et États-Unis). L'étude se composait de deux parties, une étude longitudinale au cours de laquelle des sujets ont été suivis de la naissance à l'âge de deux ans, et une étude transversale d'enfants âgés de 1,5 à 5 ans. Il faut noter que, comme toutes les autres courbes de croissance, les normes de l'OMS correspondent à la taille en décubitus de la naissance à deux ans puis en position debout par la suite.

\section{Étude longitudinale}

Sur les 1737 sujets inclus dans l'étude longitudinale, 882 l'ont terminée et seules leurs données ont été utilisées. Des nourrissons nés à terme ont été inclus sans tenir compte de leur poids de naissance de telle sorte que l'échantillon comportait $2,3 \%$ de nourrissons de poids de naissance $<2500$ g. Les enfants ont été alimentés conformément aux recommandations de l'OMS, donc allaités au sein jusqu'à l'âge d'un an avec introduction d'aliments complémentaire uniquement après l'âge de six mois. Leurs mères étaient non fumeuses. Les critères d'éligibilité ont été stricts dans certains pays, aboutissant à la non-inclusion de la majorité des sujets des tranches respectives, alors que dans d'autres, ils n'ont entraîné la non-inclusion que d'une petite proportion de sujets. Ces critères signifiaient également que les sujets provenaient généralement de couches sociales plus privilégiées.

\section{Étude transversale}

Les sujets ont été recrutés dans les mêmes couches démographiques que ceux inclus dans l'étude longitudinale. Les 6 669 sujets âgés de 18 à 71 mois ont tous été mesurés une fois. En raison des effets nocifs du surpoids, les données des sujets dont le poids pour la taille était supérieur à deux écarts types n’ont pas été incluses, ce qui a amené à la non-inclusion de 1,4\% des garçons et de $1,1 \%$ des filles. 


\section{Autres courbes de croissance}

Les courbes de croissance de 1977 du NCHS [1] allant de la naissance à trois ans reposaient sur des données longitudinales recueillies par l'Institut Fels de 1929 à 1975 [8]. Les sujets étaient majoritairement blancs et vivaient dans une zone géographique limitée des États-Unis. La majorité d'entre eux avaient été allaités au sein, mais certains ne l'avaient été que pendant une courte durée, et une minorité importante ne l'avait pas été du tout. Le poids et la taille avaient été mesurés tous les trois mois au cours de la première année de la vie. Pour les âges de deux à 18 ans, le NCHS s'était appuyé sur des données transversales représentatives provenant d'enquêtes sanitaires nationales.

Les courbes des CDC de 2000 [6, 7] sont une modification des courbes du NCHS. De la naissance à trois ans, les données longitudinales originales ont été remplacées par les données transversales provenant de cinq enquêtes représentatives au plan national menées aux États-Unis de 1963 à 1994. En raison de la rareté des données sur les nourrissons, des données supplémentaires pour la taille (mais non pour le poids) provenant d'établissement de soins soutenus par le gouvernement (Women, Infants and Children) ont été utilisées. Dans l'ensemble, les données sur le poids n'ont été disponibles que pour moins de 300 sujets par mois (garçons et filles) de 3 à 12 mois (aucune donnée sur le poids ne l'étant pour les 2 premiers mois). Les données sur la taille ont été disponibles pour un grand nombre de sujets âgés de 1 à 5 mois, mais pour moins de 300 par mois entre 6 et 12 mois. En raison du nombre limité de sujets, la confiance en la précision des percentiles extrêmes est faible. Aucune information sur la nutrition des nourrissons n'est disponible. Les données sur le poids des enfants âgés de plus de six ans obtenues de 1988 à 1994 n'ont pas été incluses en raison de la prévalence élevée de poids «non sains» (élevés) dans cette cohorte.

Courbe Euro-Growth de 2000 [5]. Les données destinées à la création des courbes de croissance Euro-Growth ont été recueillies dans 22 centres de 11 pays européens. Des sujets nés entre 1990 et 1993 ont été longitudinalement suivis de la naissance à cinq ans. Sur les 2245 sujets inclus, 1746 ont terminé l'étude jusqu'à l'âge de 12 mois, 1205 jusqu'à l'âge de 24 mois et 1071 jusqu'à l'âge de trois ans. Les sujets étaient nés à au moins 37 semaines de gestation et leur poids de naissance était supérieur à $2500 \mathrm{~g}$. Une minorité de sujets était alimentée conformément aux recommandations de l'OMS (allaitement maternel jusqu'à l'âge d'un an, aliments complémentaires uniquement après l'âge de six mois).

\section{Évaluation fonctionnelle}

L'impact fonctionnel des différences entre courbes a été évalué à l'aide d'échantillons hypothétiques. La distribution des poids et des rapports taille/poids était normale dans ces échantillons, qui étaient centrés sur le $50^{\text {ème }}$ percentile des courbes du NCHS.

Les nouvelles normes de croissance de l'OMS

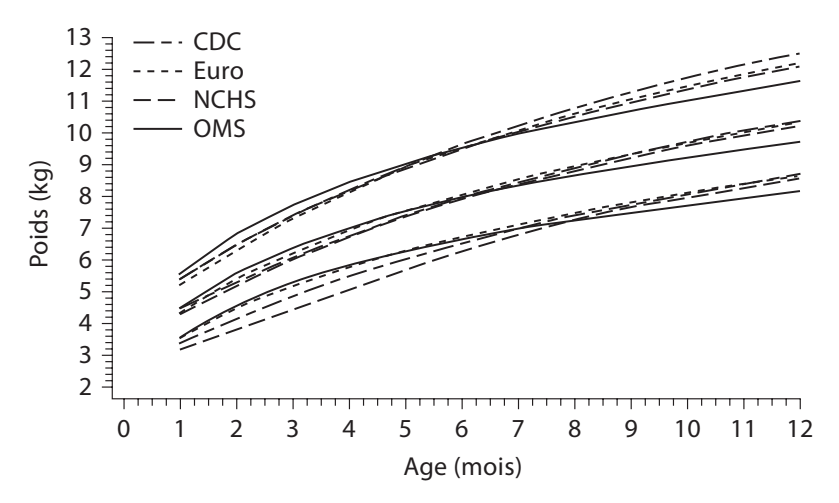

Fig. 1. Poids pour l'âge des nourrissons de sexe masculin âgés de 1 à 12 mois. Les $5^{\text {ème }}, 50^{\text {ème }}$ et $95^{\text {ème }}$ percentiles des normes de l'OMS sont présentés comparativement à ceux des courbes EuroGrowth, du NCHS et des CDC.

\section{Examen comparatif des normes de l'OMS}

Première année de la vie

La figure 1 montre les $5^{\text {ème }}, 50^{\text {ème }}$ et $95^{\text {ème }}$ percentiles du poids pour la taille chez les garçons au cours de la première année de la vie selon les normes de l'OMS comparativement aux trois autres courbes. (Les relations entre les courbes sont dans tous les cas similaires pour les filles, dont les courbes ne sont pas présentées séparément.) Il est évident que, au cours des six premiers mois, les poids indiqués par les références de l'OMS tendent à être plus élevés que ceux mentionnés par les autres courbes à tous les percentiles. L'inverse apparaît au cours du deuxième semestre de la vie, le poids de référence de l'OMS étant progressivement plus bas que celui indiqué par les autres courbes. L'ordre de grandeur des différences n'est pas le même pour tous les percentiles. Les différences sont particulièrement importantes pour le $5^{\text {ème }}$ percentile au cours des six premiers mois, alors qu'elles sont plutôt homogènes pendant le second semestre. Afin de faciliter la comparaison, la figure 2 montre le $50^{\text {ème }}$ percentile des autres courbes exprimé en unités d'écart type (score z) des normes de l'OMS. Les différences atteignent 0,3-0,6 unité d'écart type au cours du premier semestre. Après 6 mois, elles atteignent environ 0,5 unité d'écart type à 12 mois, mais dans le sens opposé.

Pour la taille (fig. 3), les différences entre les normes de l'OMS et les autres courbes sont moins spectaculaires que pour le poids. L'écart entre les percentiles externes tend à être plus étroit pour les normes de l'OMS que pour les autres courbes. Comme le montre la figure 4 , les différences au $50^{\text {ème }}$ percentile tendent à être inférieures à 0,3 unité d'écart type. Contrairement au poids indiqué par les courbes des CDC, la taille mentionnée par ces mêmes courbes diffère fortement de celle citée par les courbes du NCHS et Euro-Growth. Aucune explication de cette différence n'a été apportée.

Ann Nestlé [Fr] 2007;65:111-117 


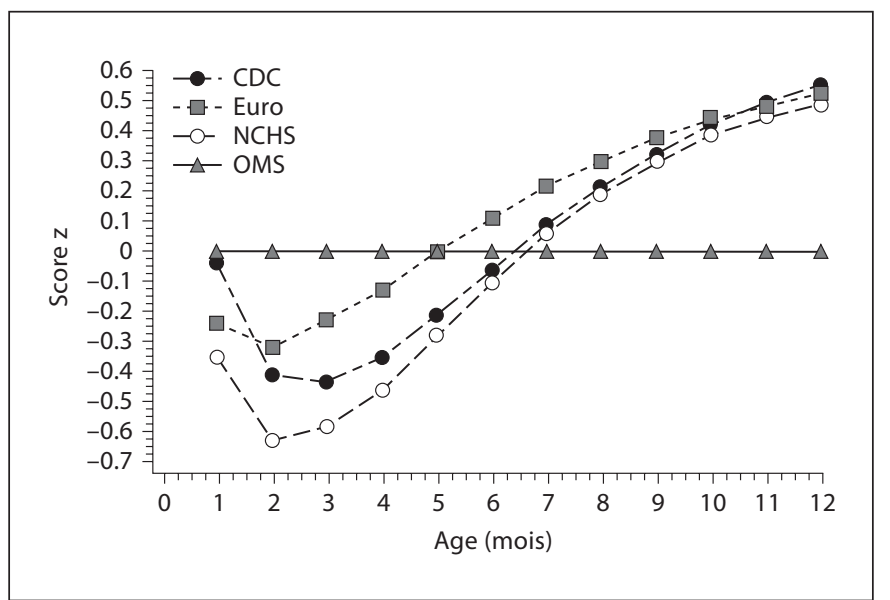

Fig. 2. $50^{\text {ème }}$ percentile du poids pour l'âge (nourrissons des deux sexes) des courbes Euro-Growth, des CDC et du NCHS exprimé en scores $\mathrm{z}$ des normes OMS.

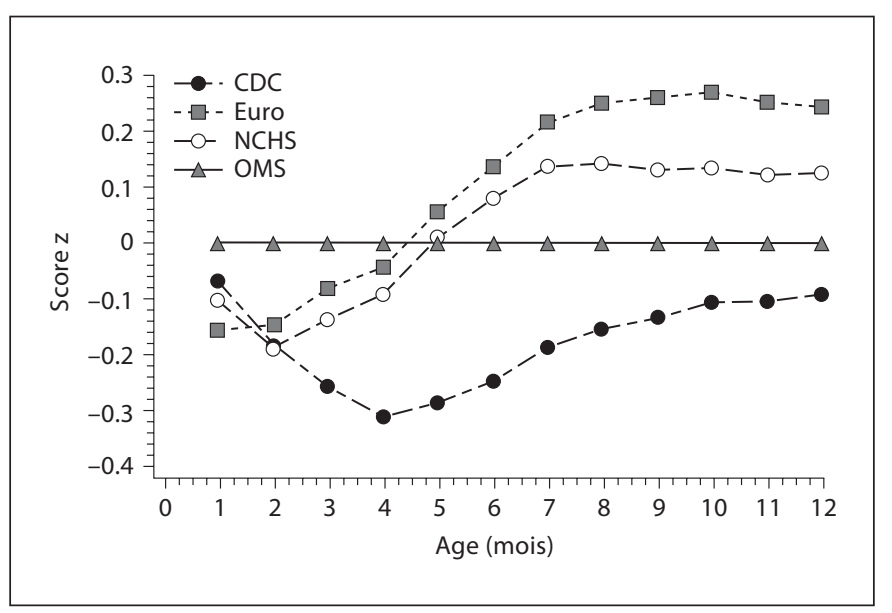

Fig. 4. $50^{\text {ème }}$ percentile de la taille pour l'âge (nourrissons des deux sexes) des courbes Euro-Growth, des CDC et du NCHS exprimé en scores $\mathrm{z}$ des normes OMS.

Des données publiées $[2,9,10]$ et non publiées sur la croissance d'enfants au sein ou nourris par formules ont été utilisées afin de tenter d'élucider l'origine des différences entre les courbes. Une comparaison avec ces données (non présentée) a permis de conclure que les normes de l'OMS et les courbes Euro-Growth représentaient plus fidèlement la croissance au cours du premier semestre de la vie que les courbes du NCHS et des CDC. Les différences de poids entre les nourrissons allaités au sein et nourris par formules sont beaucoup

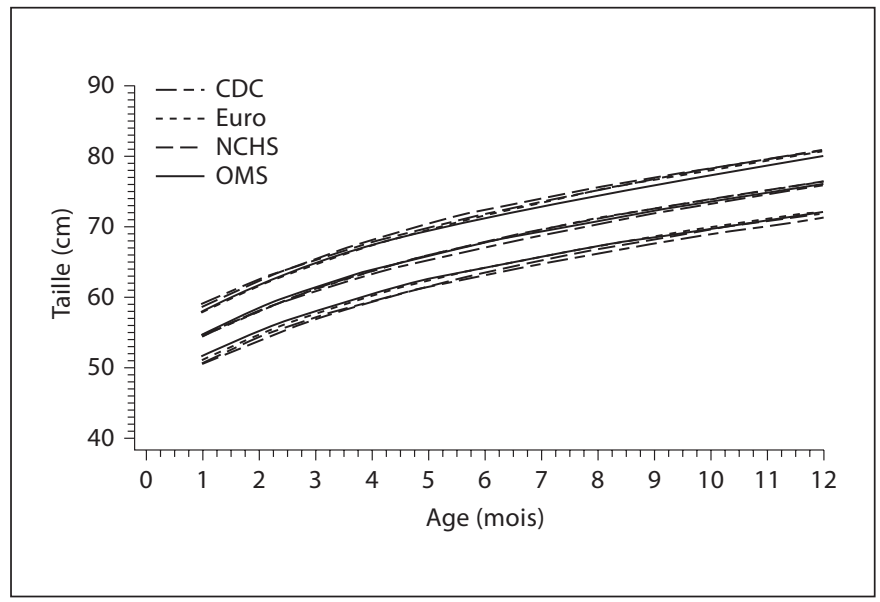

Fig. 3. Taille pour l'âge des nourrissons de sexe masculin âgés de 1 à 12 mois. Les $5^{\text {ème }}, 50^{\text {ème }}$ et $95^{\text {ème }}$ percentiles des normes de l'OMS sont présentés comparativement à ceux des courbes EuroGrowth, du NCHS et des CDC.

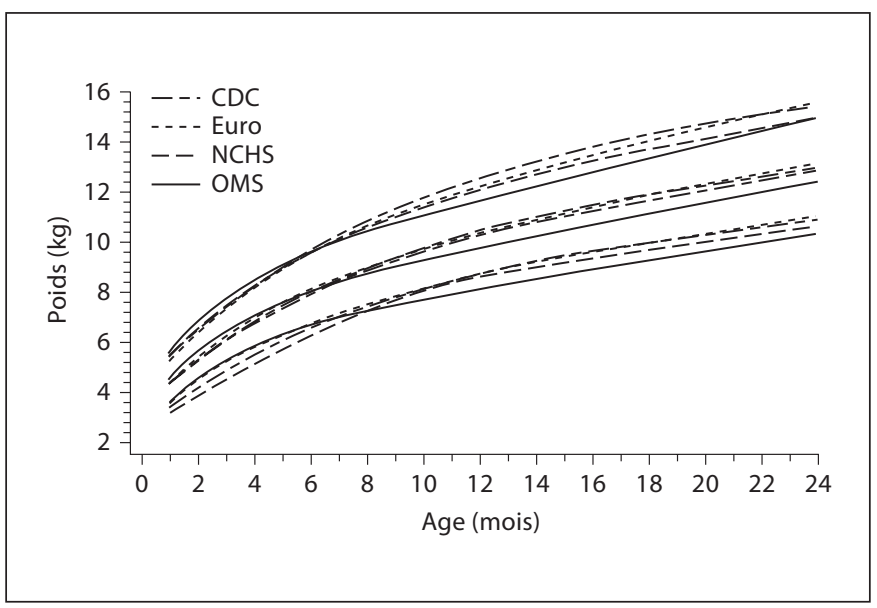

Fig. 5. Poids pour l'âge des enfants de sexe masculin âgés de 12 à 24 mois. Les $5^{\text {ème }}, 50^{\text {ème }}$ et $95^{\text {ème }}$ percentiles des normes de l'OMS sont présentés comparativement à ceux des courbes Euro-Growth, du NCHS et des CDC.

plus petites que celles constatées entre les normes de l'OMS d'une part et les courbes du NCHS et des CDC d'autre part. Cette observation a permis de conclure de plus que les inconvénients des courbes du NCHS, et également de celles du CDC, au cours des six premiers mois de la vie ne traduisaient pas des différences de régime alimentaire (allaitement au sein ou nutrition par formules) mais étaient dus à d'autres causes, par exemple au nombre restreint de mesures et, probablement, aux méthodes d'ajustement et de lissage des courbes. 


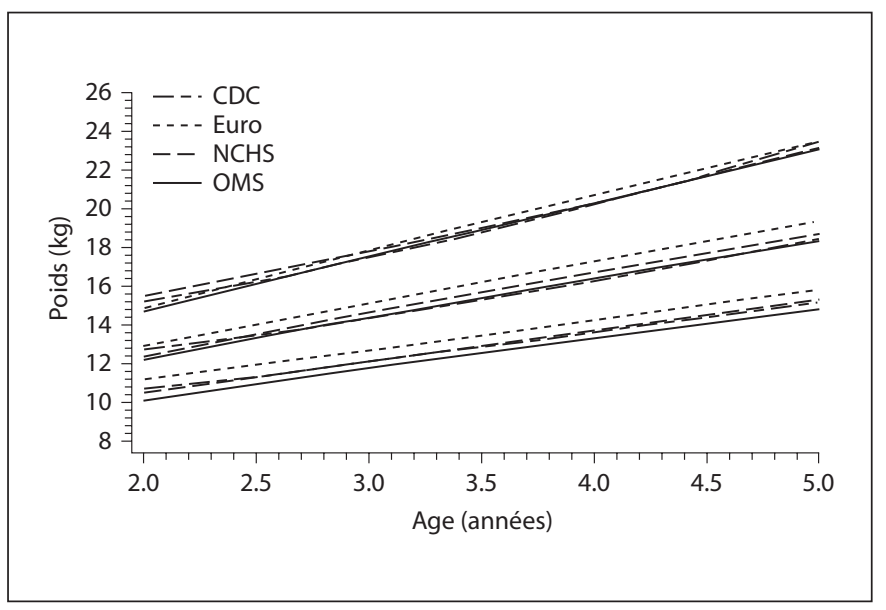

Fig. 6. Poids pour l'âge des enfants de sexe masculin âgés de 2 à 5 ans. Les $5^{\text {ème }}, 50^{\text {ème }}$ et $95^{\text {ème }}$ percentiles des normes de l'OMS sont présentés comparativement à ceux des courbes Euro-Growth, du NCHS et des CDC.

Ces comparaisons ont également permis de conclure que les courbes des CDC ne représentaient pas une amélioration substantielle par rapport aux inadéquations des courbes antérieures du NCHS. Les courbes du NCHS et des CDC ne représentent pas très correctement la croissance au cours du premier semestre de la vie.

Deuxième année de la vie

Les tendances et différences apparaissant pendant le second semestre de la vie persistent au cours de la deuxième année. Comme le montre la figure 5 , tous les percentiles du poids des normes de l'OMS sont plus bas que ceux des autres courbes. De même, les percentiles de taille (non présentés) poursuivent les trajectoires établies de 6 à 12 mois, les normes de l'OMS continuant à présenter une moindre dispersion entre les percentiles externes que celle indiqué par les autres courbes.

\section{De deux à cinq ans}

D'importantes différences concernant le poids (fig. 6) et la taille (fig. 7) apparaissent entre les courbes concernant les âges de deux à cinq ans. Le poids mentionné par les courbes EuroGrowth (mais non la taille) tend à être plus élevé que celui indiqué par les autres courbes, avec une marge considérable $(0,4-0,6$ unité d'écart type), ce qui est difficile à expliquer. Les percentiles de poids des normes de l'OMS tendent à occuper l'extrémité inférieure du spectre. Ce fait s'explique, au moins pour les percentiles supérieurs, par la non-inclusion des données de certains des enfants les plus lourds. Le profil particulier des poids indiqués par les courbes des CDC des âges de deux à trois ans résulte de la liaison entre les courbes des nourrissons et celles des enfants.

Les nouvelles normes de croissance de l'OMS

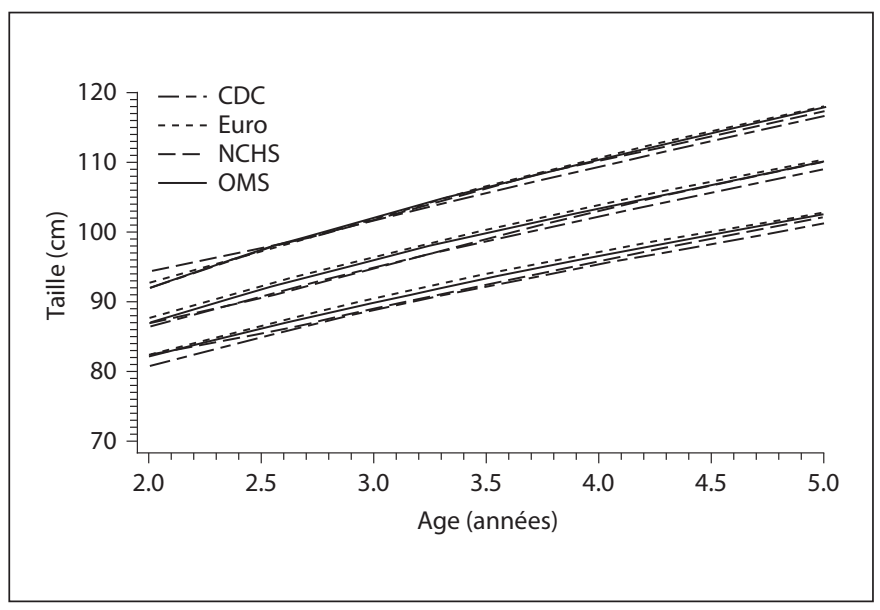

Fig. 7. Taille pour l'âge des enfants de sexe masculin âgés de 2 à 5 ans. Les $5^{\text {ème }}, 50^{\text {ème }}$ et $95^{\text {ème }}$ percentiles des normes de l'OMS sont présentés comparativement à ceux des courbes Euro-Growth, du NCHS et des CDC.

\section{Évaluation fonctionnelle}

La figure 8 compare les pourcentages d'enfants en bonne santé âgés d'un à deux ans identifiés comme étant d'un poids insuffisant (poids $<10^{\text {ème }}$ percentile). Ce pourcentage est nettement plus bas avec les normes de l'OMS qu'avec les autres courbes, bien que les différences entre ces dernières soient également substantielles. Avec les normes de l'OMS, environ 3\% des enfants sont identifiés comme ayant un poids insuffisant, alors que cette proportion est d'environ $8 \%$ avec les courbes du NCHS et d'environ 13-14\% avec les courbes du CDC et EuroGrowth. On peut concevoir que l'utilisation des nouvelles normes de l'OMS puisse aboutir à l'identification retardée d'un retard de croissance chez les nourrissons et les jeunes enfants nourris artificiellement.

La figure 9 présente un autre exemple. Chez les garçons en bonne santé âgés de deux à cinq ans, la proportion de ceux identifiés comme étant en surpoids (IMC $>90^{\text {ème }}$ percentile) est d'environ $16 \%$ avec les références de l'OMS, mais n'est que d'environ $11 \%$ avec les courbes des CDC et d'environ $7 \%$ avec les courbes Euro-Growth.

Ces exemples illustrent les différences notables entre les proportions des enfants identifiés quand les différentes courbes sont utilisées à des fins de dépistage. Il existe des différences entre toutes les courbes, mais, pour certaines des variables les plus importantes (fig. 8, 9), les références de l'OMS sont à l'extrême du spectre. Cette situation résulte largement de l'effet voulu de l'approche normative appliquée par l'OMS.

Ann Nestlé [Fr] 2007;65:111-117 


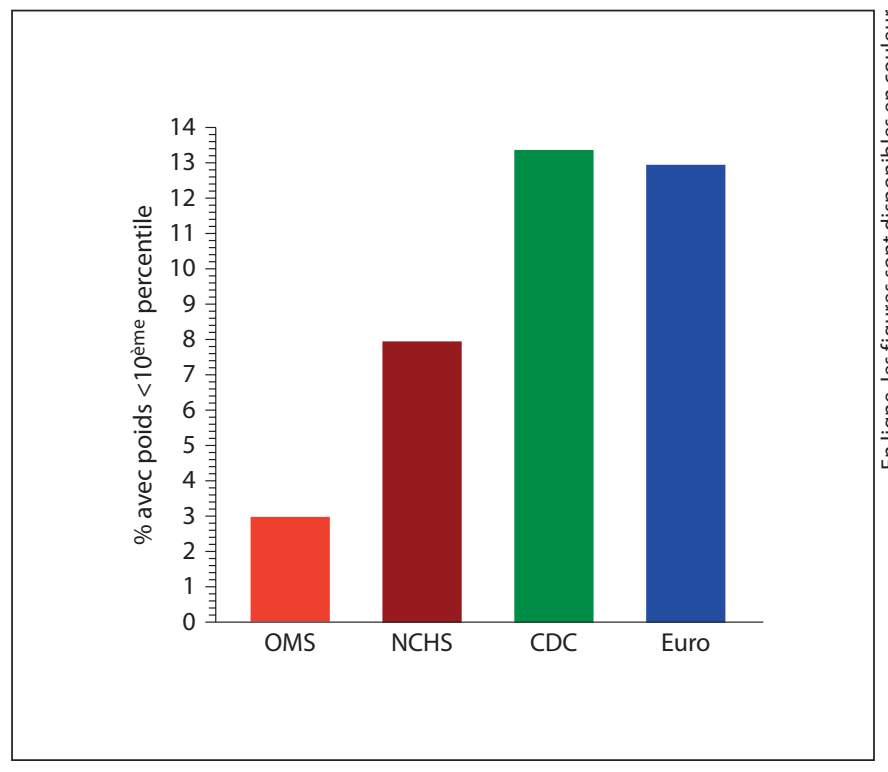

Fig. 8. Pourcentage des enfants de 1 à 2 ans en bonne santé identifiés comme d'un poids insuffisant au moyen des courbes de l'OMS, du NCHS, des CDC et Euro-Growth.

\section{Commentaires}

Les nouvelles normes de croissance de l'OMS ont été conçues selon une approche normative et visent à décrire la croissance des enfants lors de son déroulement optimal, sans obstacle dû à des facteurs externes. Ces normes diffèrent fortement des courbes de croissance de référence existantes. Il existe de nombreuses différences entre les courbes existantes, mais les nouvelles normes de l'OMS se singularisent d'ellesmêmes en suivant un profil différent. Cette situation résulte largement de l'approche normative. Les différences sont loin d'être uniformes, changeant de direction et d'ordre de grandeur avec l'âge. Par exemple, au cours du premier semestre de la vie, le poids indiqué par les normes de l'OMS est plus élevé que celui mentionné par toutes les autres courbes, alors que la situation inverse s'observe au cours du second semestre. Les différences de poids au cours des six premiers mois ne s'expliquent pas totalement par des différences de régime alimentaire, et résultent partiellement d'autres facteurs, la plupart de nature technique.

Pour l'utilisation des courbes lors de l'évaluation d'un enfant donné (utilisation clinique), les conséquences de leurs différences sont probablement mineures. Après tout, le clinicien évalue habituellement la croissance sur la base de plusieurs mesures et tend à la juger par le degré du parallélisme de son déroulement par rapport aux percentiles. De ce fait, pour une utilisation clinique, la position des percentiles est moins importante que leur direction. D’autre part, pour l'utilisation épi-

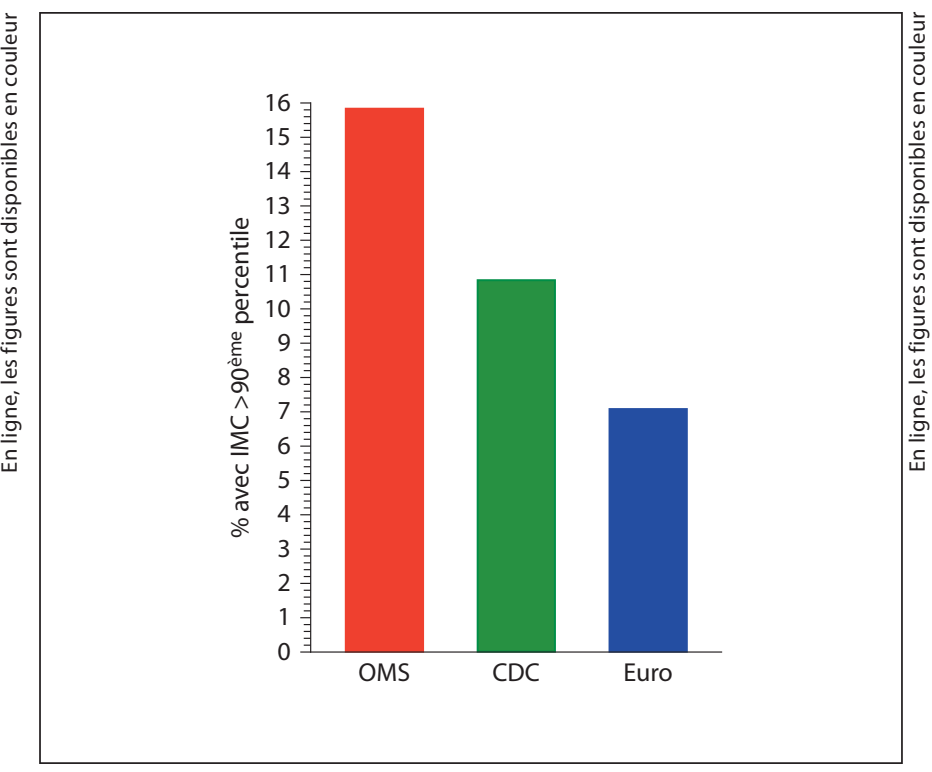

Fig. 9. Pourcentage des enfants de 2 à 5 ans en bonne santé identifiés comme en surpoids au moyen des courbes de l'OMS, des CDC et Euro-Growth.

démiologique des courbes de croissance, dont l'objectif est de déterminer la prévalence des croissances aberrantes, la position des percentiles externes est du plus grand intérêt. Ici, l'impact des différences entre les courbes de croissance peut être considérable. Comme les exemples des figures 8 et 9 l'illustrent, la proportion des sujets identifiés par les diverses courbes varie fortement. Cet effet est intentionnel dans le cas des normes de croissance de l'OMS. L'objectif de ces normes est par exemple l'identification d'un plus grand nombre d'enfants en surpoids qu'avec les autres courbes, sans doute parce que cela permettrait de mieux attirer l'attention sur le problème du surpoids infantile. De ce fait, des corrections visant à revenir aux seuils traditionnels iraient à l'encontre des intentions des normes de croissance de l'OMS.

\section{Références}

1 Hamill PV, Drizd TA, Johnson CL, Reed RB, Roche AF: NCHS growth curves for children birth-18 years. United States. Vital Health Stat 11 1977; 165:i-iv, 1-74.

2 Dewey KG, Peerson JM, Brown KH, Krebs NF, Michaelsen KF, Persson LA, Salmenpera L, Whitehead RG, Yeung DL, WHO Working Group on Infant Growth: Growth of breast-fed infants deviates from current reference data: a pooled analysis of US, Canadian and European data sets. Pediatrics 1995;96:495-503. 
3 de Onis M, Habicht JP: Anthropometric reference data for international use: recommendations from a World Health Organization Expert Committee. Am J Clin Nutr 1996;64:650-658.

4 WHO Multicentre Growth Reference Study Group: WHO child growth standards based on length/height, weight and age. Acta Paediatr Suppl 2006;450:76-85.

5 Haschke F, van t'Hoff MA (eds): EuroGrowth. J Pediatr Gastroenterol Nutr 2000; 31(suppl 1):S1-S86.
6 KuczmarskiRJ, Ogden CL, Grummer-Strawn LM, Flegal KM, Guo SS, Wei R, Mei Z, Roche AF, Johnson CL: CDC growth charts: United States. Adv Data 2000;314:1-27.

7 Ogden CL, Kuczmarski RJ, Flegal KM, Mei Z, Guo S, Wei R, Grummer-Strawn LM, Curtin LR, Roche AF, Johnson CL: Centers for Disease Control and Prevention 2000 growth charts for the United Sates: improvements to the 1977 National Center for Health Statistics version. Pediatrics 2002;109:45-60.
8 Roche AF: Growth, Maturation and Body Composition: The Fels Longitudinal Study 1929-1991. Cambridge, Cambridge University Press, 1992.

9 Nelson SE, Rogers RR, Ziegler EE, Fomon SJ: Gain in weight and length during early infancy. Early Hum Dev 1989;19:223-239.

10 Haschke F, van t'Hoff MA; Euro-Growth Study Group: Euro-Growth references for breast-fed boys and girls: influence of breastfeeding and solids on growth until 36 months of age. J Pediatr Gastroenterol Nutr 2000; 31(suppl 1):S60-S71. 